(C) by Oldenbourg Wissenschaftsverlag, München

\title{
Point groups in crystallography
}

\author{
Massimo Nespolo*,I and Bernd Souvignier ${ }^{\mathrm{II}}$ \\ I Nancy-Université, Faculté des Sciences et Techniques, Cristallographie, Résonance Magnetique et Modélisations (CRM2), \\ UMR - CNRS 7036, Boulevard des Aiguillettes, BP 70 239, 54506 Vandœvre-lès-Nancy cedex, France \\ II Radboud University, Faculty of Science, Mathematics and Computing Science, Institute for Mathematics, Astrophysics and Particle Physics. \\ Postbus 9010, 6500 GL Nijmegen, The Netherlands
}

Received July 3, 2008; accepted September 16, 2008

\section{Geometric crystal class / Point group / Point space / Site-symmetry group / Vector space / Affine space / Factor group}

Abstract. Two dual spaces are extensively used in crystallography: the point space $E^{n}$, hosting the crystal pattern; and the vector space $\boldsymbol{V}^{n}$, where face normals and reciprocal-lattice vectors are defined. The term "point group" is used in crystallography to indicate four different types of groups in these two spaces.

1) Morphological point groups in $\boldsymbol{V}^{n}$; they can be obtained by determining subgroups of maximal holohedries (holohedries not in group-subgroup relation): this gives 21 and 136 point groups in $V^{2}$ and $V^{3}$, respectively, which are classified into 10 and 32 pointgroup types (on the basis of which geometric crystal classes are defined) falling into 9 and 18 abstract isomorphism classes.

2) Symmetry groups of atomic groups and coordination polyhedra in $E^{n}$; they coincide with molecular point groups, which are infinite in number because the symmetry operations forming these groups are not subject to the crystallographic restriction.

3) Site-symmetry groups in $E^{n}$; they are finite groups but infinite in number due to conjugation by the translation subgroups of the space groups. They are classified in geometric crystal classes exactly like point groups in $\boldsymbol{V}^{n}$. A finer classification of site-symmetry groups into species is however introduced that takes into account their orientation in space: species of site-symmetry groups in $E^{n}$ uniquely correspond to point groups in $\boldsymbol{V}^{n}$.

4) Groups of matrices representing the linear parts of space group operations in $E^{n}$; they are isomorphic to the point groups in $\boldsymbol{V}^{n}$ and are also isomorphic to the factor groups $\mathcal{G} / \mathcal{T}$, where $\mathcal{G}$ is a space group and $\mathcal{T}$ its translation subgroup.

* Correspondence author (e-mail: massimo.nespolo@crm2.uhp-nancy.fr)

\section{Introduction}

Point groups in chemistry are defined in an unambiguous way as the symmetry groups of molecules. Due to the finite number of atoms in a molecule, all operations in the symmetry group leave fixed at least one point, namely the center of the atom positions. When the concept of symmetry groups with a fixed point is applied to crystals, the periodicity of the atomic distribution enforces the well-known restriction that, in the physical three-dimensional space, the order of a rotation is $2,3,4$ or 6 . This is often the only difference that comes to mind when going from molecular to crystallographic point groups. But taking a closer look, the concept of point groups in crystallography turns out to be far more complex, as we are going to show.

The International Tables for Crystallography, Volume A ([7], ITA in the sequel of this paper; page numbers are given with reference to the 5th Edition, 2002) distinguish three different types of crystallographic point groups (see also [13]):

1) the point group $\mathcal{P}$ of the crystal, as the symmetry group of the vector set of face normals representing the external shape (morphology) of a macroscopic crystal and its physical properties (pages 725, 762) ITA);

2) the symmetry groups of atomic groups and coordination polyhedra (page 762);

3 ) the site-symmetry groups $S(X)$, as the set of all symmetry operations of a space group $\mathcal{G}$ that leave a point $X$ invariant (pages 732, 762);

4) the groups of matrices representing the linear parts of space group symmetry operations (page 724).

The groups referred to in no. 1) and no. 4) are isomorphic but are defined in two dual spaces, whereas the difference between no. 2) and no. 3) concerns mainly the objects on which the groups act, namely finite polyhedra in crystals vs. infinite crystal patterns ${ }^{l}$.

Note that the term point group is appropriate in all cases, since the groups in question all leave some "point" fixed (which may in fact be a vector rather than a point, as will be explained below).

1 A crystal pattern is the idealization of a crystal structure to any pattern (not necessarily atomic) having a crystalline symmetry and periodicity. 


\section{Dual spaces in crystallography}

An $n$-dimensional space is commonly thought of as the space of columns with $n$ real components, indicated by $\mathbb{R}^{n}$. The ITA, however, makes extensive use of two different spaces of dimension $n$, the point space $E^{n}$ ( $E$ for Euclidean) and the vector space $\boldsymbol{V}^{n}$. The former is the space where atoms exist, the latter the space where face normals and reciprocal-lattice vectors are defined. From the mathematical viewpoint, $E^{n}$ is an affine space, while $\mathbf{V}^{n}$ is a vector space sensu strictu (see also [11]).

The vector space $\boldsymbol{V}^{n}$, however, occurs in two disguises. Regarded as the vector space containing face normals and reciprocal-lattice vectors, its elements are commonly written with respect to a basis $\left(\boldsymbol{a}_{1}{ }^{*}, \ldots, \boldsymbol{a}_{\mathrm{n}}{ }^{*}\right)$ of $\boldsymbol{V}^{n}$. But in a second interpretation, $\boldsymbol{V}^{n}$ is the vector space underlying the point space $E^{n}$ in which for example the translation vectors live. Vectors in this vector space are commonly written with respect to a basis $\left(\boldsymbol{a}_{1}, \ldots, \boldsymbol{a}_{n}\right)$. Since the two bases $\left(\boldsymbol{a}_{1}, \ldots, \boldsymbol{a}_{n}\right)$ and $\left(\boldsymbol{a}_{1}{ }^{*}, \ldots, \boldsymbol{a}_{n}{ }^{*}\right)$ of $\mathbf{V}^{n}$ are commonly chosen such that $\boldsymbol{a}_{i} \cdot \boldsymbol{a}_{j}{ }^{*}=0$ for $i \neq j$, i.e. as dual bases (up to scalars), the two instances of the vector space $V^{n}$ can be thought of as two dual spaces ${ }^{2}$. By a slight abuse of language, the spaces $E^{n}$ (identified with its underlying vector space) and $\boldsymbol{V}^{n}$ (in its interpretation as containing face normals) may therefore be termed two dual spaces.

In order to clarify the concepts underlying the point and vector spaces, we will briefly review the general definitions of vector spaces and affine spaces.

An abstract vector space consists of a set of vectors for which vector addition and multiplication by scalars from a field $^{3} F$ are defined. In crystallography, $F$ is almost always the field $\mathbb{R}$ of real number, occasionally the field $\mathbb{C}$ of complex numbers and rarely the field $\mathbb{Q}$ of rational numbers.

A set $\boldsymbol{V}$ of vectors is called a vector space over the field $F$ if the two binary operations

1. vector addition: $\boldsymbol{v}+\boldsymbol{w} \in \boldsymbol{V}$ for $\boldsymbol{v}, \boldsymbol{w} \in \boldsymbol{V}$;

2. scalar multiplication: $a \boldsymbol{v} \in \boldsymbol{V}$ for $a \in F$ and $\boldsymbol{v} \in \boldsymbol{V}$ satisfy the following eight axioms:

1. Vector addition is associative: $\boldsymbol{u}+(\boldsymbol{v}+\boldsymbol{w})=(\boldsymbol{u}+\boldsymbol{v})$ $+\boldsymbol{w}$

2. Vector addition is commutative: $\boldsymbol{v}+\boldsymbol{w}=\boldsymbol{w}+\boldsymbol{v}$;

3. Vector addition has an identity element, the zero vector $\mathbf{0}: \boldsymbol{v}+\mathbf{0}=\mathbf{0}+\boldsymbol{v}=\boldsymbol{v}$

4. Vector addition has inverse elements: for every $\boldsymbol{v} \in \boldsymbol{V}$ there exists $-\boldsymbol{v} \in \boldsymbol{V}$ such that $\boldsymbol{v}+(-\boldsymbol{v})=0$;

5. Distributivity holds for scalar multiplication over vector addition: $a(\boldsymbol{v}+\boldsymbol{w})=a v+a w$;

6. Distributivity holds for scalar multiplication over field addition: $(a+b) v=a v+b v$;

${ }^{2}$ Note that in a strict mathematical sense the dual space of a vector space $\boldsymbol{V}$ is the space of linear mappings from $\boldsymbol{V}$ to $\mathbb{R}$. However, in the presence of a scalar product, a linear mapping $l: V \rightarrow \mathbb{R}$ can be identified with a uniquely determined vector $\boldsymbol{w} \in \boldsymbol{V}$ such that $l(\boldsymbol{v})=\boldsymbol{v} \cdot \boldsymbol{w}$ for all $\boldsymbol{v} \in \boldsymbol{V}$.

${ }^{3}$ A field is an algebraic structure in which the operations of addition, subtraction, multiplication and division (except division by zero) may be performed, and where the common rules of the arithmetic (commutativity, associativity, distributivity) hold.
7. Scalar multiplication is compatible with multiplication in the field of scalars: $a(b v)=(a b) v$;

8. Scalar multiplication has an identity element: $1 \boldsymbol{v}=\boldsymbol{v}$, where 1 denotes the multiplicative identity in $F$.

Among the vectors of a vector space, the zero vector $\mathbf{0}$ is distinguished as the identity element for vector addition. It plays the role of origin in the vector space.

From a vector space $\boldsymbol{V}$, a corresponding affine space $A$ can be obtained, whose elements are points and in which two points differ by a unique vector in $\boldsymbol{V}$.

Clearly, one space can be easily derived from the other: the vector space $\boldsymbol{V}$ is the set of difference vectors between pairs of points of $A$ and conversely the affine space is built by translating a single (but arbitrary) point of $A$ by each of the vectors in $\boldsymbol{V}$. The crucial difference is that in contrast to the zero vector in $\boldsymbol{V}$ no point is in principle distinguished in the affine space $A$. Only when the affine space is "decorated", which in our case means occupied by a crystal pattern and its underlying lattice, the points lose their equal status. Not every point will then be considered as a possible origin.

After choosing an origin $O$, the points of the affine space are in 1-1 correspondence with the vectors of $\boldsymbol{V}$ by identifying a point $P$ with the difference vector $\overrightarrow{\mathrm{OP}}$. This results in the well-known direct space, whose elements connect each point of point space with the origin $O$ and whose basis vectors are indicated as $\boldsymbol{a}_{i}(i=1, \ldots, n)$. Combined with the origin, this basis forms the coordinate system $\left(O, \boldsymbol{a}_{1}, \ldots, \boldsymbol{a}_{n}\right)$ of the point space $E^{n}$ of ITA.

Because two parallel vectors of the same length are actually the same vector in the vector space, each lattice row in point space is represented by a single vector in vector space, namely the vector connecting two neighbouring nodes in the lattice row.

A lattice plane in point space is represented by two (linearly independent) vectors in vector space. However, in the case of 3-dimensional space, also a lattice plane can be represented by a single vector in vector space: taking any two vectors $\boldsymbol{v}, \boldsymbol{w}$ in $\boldsymbol{V}^{3}$ which represent a lattice plane in $E^{3}$, their vector product $\boldsymbol{v} \times \mathbf{w}$ is still a vector in $\boldsymbol{V}^{3}$ which now is perpendicular to the plane in $E^{3}$. Its length and orientation, however, depend on the vectors chosen to represent the lattice plane. The length of the vector perpendicular to the given lattice plane gives rise to a degree of freedom:

- If this length is taken equal to $d(h k l)$, we get Bravais' polar lattice ([1]; see also [12]), for which $\boldsymbol{a}_{i} \cdot \boldsymbol{a}_{j}{ }^{*}=V^{2 / 3} \delta_{i j}$ ( $V$ being the volume of the unit cell).

- If instead the length is taken equal to $1 / d(h k l)$, we get Gibbs' reciprocal system of vectors, for which $\boldsymbol{a}_{i} \cdot \boldsymbol{a}_{j}^{*}=\delta_{i j}$ ([5]) and on the basis of which Ewald ([4]) later introduced the reciprocal lattice ${ }^{4}$.

- For the general case of $\boldsymbol{a}_{i} \cdot \boldsymbol{a}_{j}^{*}=k \delta_{i j}$ ( $k$ being a constant), the term "pseudo-reciprocal lattice" has been introduced ([6]).

${ }^{4}$ For a lattice $L \subset V^{n}$, the reciprocal lattice $L^{*}$ of $L$ is defined by $L^{*}=\{\boldsymbol{v} \in \boldsymbol{V} \mid \boldsymbol{v} \cdot \boldsymbol{w} \in \mathbb{Z}$ for all $\boldsymbol{w} \in L\}$. If $\left(\boldsymbol{a}_{1}, \ldots, \boldsymbol{a}_{n}\right)$ is a lattice basis of $L$, then the dual basis $\left(\boldsymbol{a}_{1}{ }^{*}, \ldots, \boldsymbol{a}_{n}{ }^{*}\right)$ for which $\boldsymbol{a}_{i} \cdot \boldsymbol{a}_{j}{ }^{*}=\delta_{i j}$ is a lattice basis of $L^{*}$. 
Of fundamental importance is that both spaces are necessary to investigate the symmetry properties of the real crystal. In particular, the symmetry of the crystal pattern is defined in point space, whereas the point group of the crystal is defined in vector space. For this reason, the ITA call the latter the vector point group of the crystal (page 725).

\section{Point groups and point-group types in vector space, and the notion of geometric crystal class}

It is common knowledge that the number of point groups in the two-dimensional $\left(\boldsymbol{V}^{2}\right)$ and three-dimensional $\left(\boldsymbol{V}^{3}\right)$ vector space is 10 and 32, respectively. To each of these point groups, a geometric crystal class corresponds: this is an equivalence class in the mathematical sense, in which "objects" are collected together which share some common property.

A geometric crystal class depends on the dimension $n$ of the space, not on the type of space $\left(E^{n}\right.$ vs. $\left.V^{n}\right)$ : in fact, the same geometric crystal classes are used to categorize crystal patterns and their space groups (defined in $E^{n}$ ) as well as their physical properties (defined in $\boldsymbol{V}^{n}$ ). The information contained in the geometric crystal class is however sometimes too coarse to completely characterize the crystal properties. Donnay ([3]) criticized the use of only 32 geometric crystal classes to study the symmetry of crystals, and stated that these classes are suitable to analyse the symmetry of wooden crystal models but not that of real crystals with their underlying crystal lattices. Indeed, this was implicitly stated in the mineralogical literature well before Donnay's criticism, as shown by the classification of certain crystal forms in three (hexagonal, trigonal and tetragonal pyramids and prisms) or two (rhombohedra) orders, depending on their orientation with respect to the basis vectors (see e.g. [10]). Also the ITA (chapter $10)$, while sticking to the 32 geometric crystal classes, actually show the stereographic projections and form classification for 42 cases: not only a distinction is made for the geometric crystal classes differing in their axial setting (like $\overline{6} 2 m$ vs. $\overline{6} m 2$ and $\overline{4} 2 m$ vs. $\overline{4} m 2$ ), but trigonal geometric crystal classes are given twice (3 and $\overline{3}$ ) or three times $(32,3 m$ and $\overline{3} m$ ) because of the two possibilities for the underlying lattice, rhombohedral and hexagonal and the possible axial settings in the hexagonal case. While this is perfectly correct, it contradicts the definition of geometric crystal classes in making implicit reference to the underlying lattice. As a result, Table 10.1.2.2 (pages 770790) of ITA actually lists something between geometric and arithmetic crystal classes, the latter being obtained as combination of the geometric crystal classes with the corresponding types of Bravais lattices, giving thus 13 (in $\boldsymbol{V}^{2}$ ) and 73 (in $\boldsymbol{V}^{3}$ ) classes which in turn correspond with the symmorphic space-group types.

The number of 10 (in $\boldsymbol{V}^{2}$ ) or 32 (in $\boldsymbol{V}^{3}$ ) point groups actually refers to the types of point groups, which are defined only by the symmetry elements they contain. To each point-group type infinitely many point groups belong, depending on the choice of basis with respect to which the symmetry elements are written. But even with respect to a fixed basis, different point groups of the same type are joined into the same class, depending on the orientation in space of their symmetry directions. For example, point groups of type 2 may have rotation axes along the $a-, b$-, or $c$-axis in the orthorhombic crystal system.

In $V^{2}$ point groups are organized hierarchically in two branches: the tetragonal branch, having the holohedry $4 \mathrm{~mm}$ at its top, and the hexagonal branch, having the holohedry $6 \mathrm{~mm}$ at its top. The same holds in $\boldsymbol{V}^{3}$, where the two branches are the cubic branch, having the holohedry $m \overline{3} m$ at its top, and the hexagonal branch, having the holohedry $6 / \mathrm{mmm}$ at its top. All point groups can be derived as subgroups of members of these holohedries. The ITA show the corresponding trees of subgroups (Fig. 10.1.3.1 on page 795 and Fig. 10.1.3.2 on page 796), which actually concern point-group types and not point groups.

It is common to identify a holohedry (being a class of point groups) with the point group contained in it that is written with respect to the conventional basis. Therefore, it is reasonable to restrict oneself to the point groups written with respect to the conventional bases of the tetragonal or hexagonal holohedry in $\boldsymbol{V}^{2}$ and of the cubic or hexagonal holohedry in $\boldsymbol{V}^{3}$. These point groups represent all pointgroup types in their different orientations ${ }^{5}$.

The subgroups of the holohedries are obtained by iteratively adding symmetry operations from the holohedry as generators to the subgroups found so far, starting with the subgroups generated by a single symmetry operation. Of course, subgroups may occur more than once in this process, thus duplicates have to be disregarded. For the fairly small groups occurring as holohedries in $\boldsymbol{V}^{2}$ and $\boldsymbol{V}^{3}$ (the largest being the cubic holohedry with 48 elements) the subgroups could actually be determined by hand, but it is convenient to use a computer algebra package such as Magma ([9]) which provides powerful tools for group theoretic computations.

It is useful to collect together subgroups which are conjugate by the holohedry, because they then automatically belong to the same point-group type. Conjugacy is an equivalence relation that may both be used to classify group elements or subgroups into equivalence classes.

Two elements $p$ and $p^{\prime}$ of a point group $\mathcal{P}$ are called conjugate if there exists a conjugating element $q$ in $\mathcal{P}$ such that:

$$
q p q^{-1}=p^{\prime}
$$

The equivalence class containing the element $p$ in $\mathcal{P}$ is called the conjugacy class of $p$ and is formed by all operations $q p q^{-1}$ with $q$ running over all elements of $\mathcal{P}$.

Two subgroups $\mathcal{H}$ and $\mathcal{H}^{\prime}$ of a point group $\mathcal{P}$ are called conjugate if there exists a conjugating element $q$ in $\mathcal{P}$ such that:

$$
\left\{q p q^{-1} \mid p \in \mathcal{H}\right\}=\mathcal{H}^{\prime} \text { or in short } q \mathcal{H} q^{-1}=\mathcal{H}^{\prime} .
$$

5 One might be tempted to coin a new term for the point groups given with respect to a conventional basis, e.g. point group instances. However, since it is intuitive to work with conventional bases, we simply stick to the term point group, in line e.g. with [8], page 64 . 
From the geometric viewpoint, conjugating by an element $q$ describes a basis change and thus conjugate subgroups act essentially in the same way, although the geometric elements (axes, planes) about which the symmetry operations are applied are differently oriented in space.

\section{Subgroups of the tetragonal holohedry of $V^{2}$}

We start with the tetragonal holohedry described with respect to its conventional basis $\left(a=b ; \gamma=90^{\circ}\right)$. First taking the subgroups generated by a single one of the 8 symmetry operations and then adding a further generator to the groups thus obtained results in 10 different subgroups (including the holohedry). These groups fall into 8 conjugacy classes under the action of the holohedry $\mathcal{P}=4 \mathrm{~mm}$ (Table 1). For example, by taking $m_{[10]}$ as generator, we obtain a point group consisting of the identity and a mirror line perpendicular to the $\boldsymbol{a}$ axis. If we take this as the operation $p$ in Eq. (1) and conjugate it by all the 8 operations of $\mathcal{P}$, we obtain $m_{[10]}$ and $m_{[01]}$ (each 4 times). The groups generated by these two elements are therefore two different point groups of the same type $(m)$ which belong to one conjugacy class. If we repeat the same procedure with $p=m_{[11]}$, the result is different and consists of 4 occurrences each of $m_{[11]}$ and $m_{[1 \overline{1}]}$. These are two more different point groups, which however are again of the same type $m$ (containing only a mirror line besides the identity) as those derived before, but belong to a different conjugacy class.

If two point groups are taken from the same conjugacy class, the geometric elements about which the operations are performed are equivalent under the tetragonal holohedry, i.e. they are transformed into each other by the symmetry operations of the tetragonal holohedry. On the other hand, taking one point group of type $m$ from each of these two conjugacy

Table 1. Conjugacy classes of subgroups of the tetragonal holohedry $4 m m$ in $V^{2}$. The class type gives the short international symbol for the point-group type, order is the group order of each member of the conjugacy class, isomorphism type gives a standard notation of the abstract isomorphism type of the groups (following [2], page 345). The class length is the number of groups in a conjugacy class, generators provides generating elements for the single groups. The group no. is for convenient reference only. Horizontal lines separate the different conjugacy classes, with the exception of columns 2 and 3 , where the horizontal lines separate the abstract isomorphism types.

\begin{tabular}{l|l|l|l|l|l}
\hline $\begin{array}{l}\text { Class } \\
\text { type }\end{array}$ & Order & $\begin{array}{l}\text { Isomorphism } \\
\text { type }\end{array}$ & $\begin{array}{l}\text { Class } \\
\text { Length }\end{array}$ & Generators & $\begin{array}{l}\text { Group } \\
\text { No. }\end{array}$ \\
\hline $\mathbf{1}$ & 1 & $C_{1}$ & 1 & 1 & $\mathbf{1}$ \\
\hline $\mathbf{2}$ & 2 & $C_{2}$ & 1 & 2 & $\mathbf{2}$ \\
\cline { 1 - 4 } $\boldsymbol{m}$ & & 2 & $m_{[10]}$ & $\mathbf{3}$ \\
$m_{[01]}$ & $\mathbf{4}$ \\
\hline $\boldsymbol{m}$ & & & 2 & $m_{[11]}$ & $\mathbf{5}$ \\
\cline { 4 - 6 } & & & $C_{[11]}$ & $\mathbf{6}$ \\
\hline $\mathbf{2 m \boldsymbol { m }}$ & 4 & $C_{2} \times C_{2}$ & 1 & $m_{[10]}, 2$ & $\mathbf{7}$ \\
\hline $\mathbf{2 m \boldsymbol { m }}$ & & & 1 & $m_{[11]}, 2$ & $\mathbf{8}$ \\
\hline $\mathbf{4}$ & 4 & $C_{4}$ & 1 & 4 & $\mathbf{9}$ \\
\hline $\mathbf{4 m m}$ & 8 & $D_{4}$ & 1 & $m_{[10]}, 4$ & $\mathbf{1 0}$ \\
\hline
\end{tabular}

classes, the two groups are not conjugate under the holohedry $\mathcal{P}$ because the corresponding lattice directions (for example, [10] and [11]) are not equivalent under $\mathcal{P}$.

The fact that the four point groups in these two conjugacy classes belong to the same geometric crystal class is due to the non-crystallographic rotation by $2 \pi / 8$. This rotation is a basis change that maps the lattice direction [10] to [11] and thus conjugates the point group generated by $m_{[10]}$ into that generated by $m_{[11]}$. The rotation by $2 \pi / 8$ also causes the two subgroups of type $2 \mathrm{~mm}$ to belong to the same geometric crystal class. We therefore obtain 6 geometric crystal classes from the tetragonal holohedry.

\section{Subgroups of the hexagonal holohedry of $V^{2}$}

By proceeding in the same way as for the tetragonal holohedry, but this time with respect to the conventional hexagonal basis $\left(a=b ; \gamma=120^{\circ}\right)$ we obtain 16 subgroups of the hexagonal holohedry (including the holohedry), which fall into 10 conjugacy classes (Table 2). Five of the 16 groups have already been obtained as subgroups of the tetragonal holohedry, which leads to a total of 21 point groups in $\boldsymbol{V}^{2}$.

That some subgroups of $6 \mathrm{~mm}$ coincide with subgroups of $4 \mathrm{~mm}$ becomes clear if we use orthohexagonal axes to index the hexagonal lattice. There are three orthohexagonal cells by which the hexagonal lattice can be described, which in ITA (page 84 for the 3-dimensional case) are called $C_{1}, C_{2}$ and $C_{3}$ and have their [10] direction coincident with the hexagonal [10], [11] and [01] directions, respectively. Any of these can be chosen, for example the cell $C_{1}$ shown in the right picture of Fig. 1 (which is drawn for $E^{3}$ but applies to $E^{2}$ as well, provided the third

Table 2. Conjugacy classes of subgroups of the hexagonal holohedry $6 \mathrm{~mm}$ in $V^{2}$. Group numbers of the form " $=k$ " indicate that the respective group is equal to the subgroup no. $k$ of the tetragonal holohedry.

\begin{tabular}{|c|c|c|c|c|c|}
\hline $\begin{array}{l}\text { Class } \\
\text { type }\end{array}$ & Order & $\begin{array}{l}\text { Isomorphism } \\
\text { type }\end{array}$ & $\begin{array}{l}\text { Class } \\
\text { Length }\end{array}$ & Generators & $\begin{array}{l}\text { Group } \\
\text { No. }\end{array}$ \\
\hline 1 & 1 & $C_{1}$ & 1 & 1 & $=1$ \\
\hline 2 & \multirow[t]{3}{*}{2} & \multirow[t]{3}{*}{$C_{2}$} & 1 & 2 & $=2$ \\
\hline$m$ & & & 3 & $\begin{array}{l}m_{[10]} \\
m_{[01]} \\
m_{[11]}\end{array}$ & $\begin{array}{l}=3 \\
11 \\
12\end{array}$ \\
\hline$m$ & & & 3 & $\begin{array}{l}m_{[12]} \\
m_{[21]} \\
m_{[\overline{1} 1]}\end{array}$ & $\begin{array}{l}=4 \\
13 \\
14\end{array}$ \\
\hline 3 & 3 & $C_{3}$ & 1 & 3 & 15 \\
\hline $2 m m$ & 4 & $C_{2} \times C_{2}$ & 3 & $\begin{array}{l}m_{[10]}, 2 \\
m_{[01]}, 2 \\
m_{[11]}, 2\end{array}$ & $\begin{array}{l}=7 \\
16 \\
17\end{array}$ \\
\hline $3 m$ & \multirow[t]{2}{*}{6} & \multirow[t]{2}{*}{$D_{3}$} & 1 & $m_{[10]}, 3$ & 18 \\
\hline $3 m$ & & & 1 & $m_{[12]}, 3$ & 19 \\
\hline 6 & 6 & $C_{6}$ & 1 & 6 & 20 \\
\hline $6 \mathrm{~mm}$ & 12 & $D_{6}$ & 1 & $m_{[10]}, 6$ & 21 \\
\hline
\end{tabular}




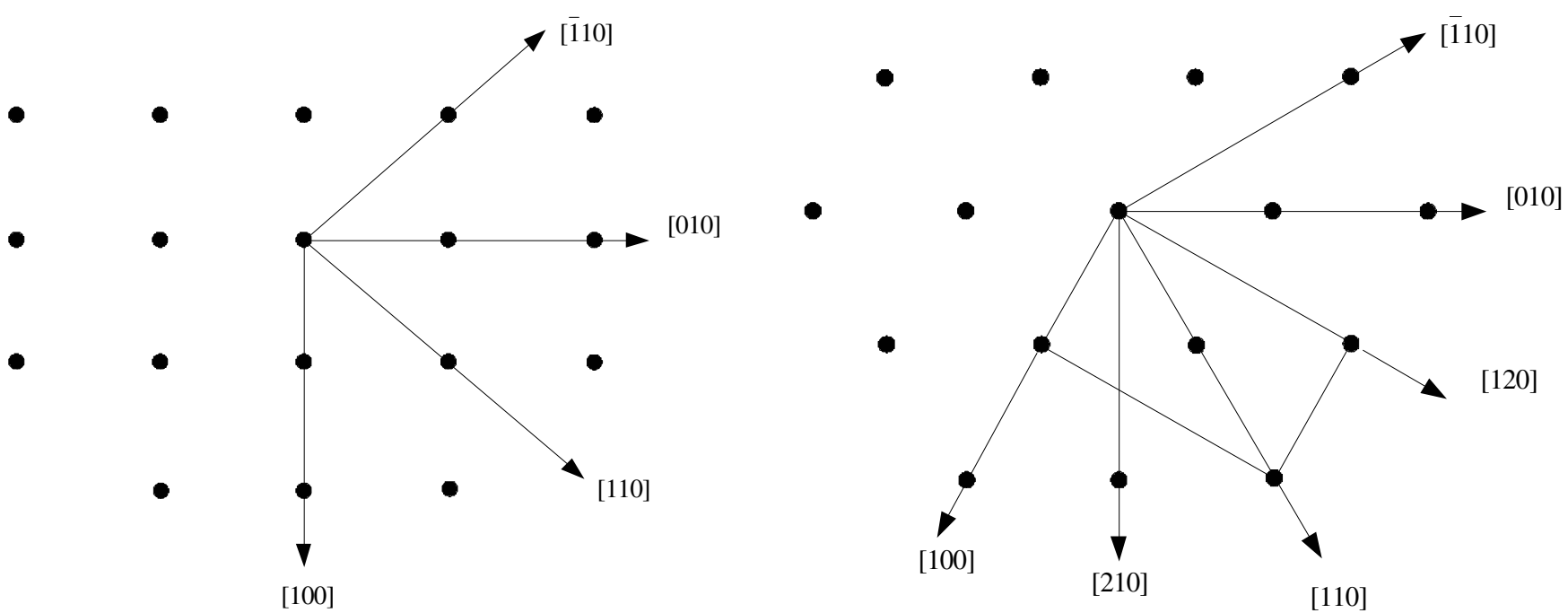

Fig. 1. Symmetry directions for the cubic (left) and hexagonal (right) lattices. In the hexagonal lattice the orthohexagonal cell $C_{1}$ is indicated, which shows the coincidence of the [120] direction in the hexagonal lattice with the [010] direction in the cubic lattice.

index of direction is neglected). In this case the [10] directions of the tetragonal and hexagonal lattices coincide and the [01] direction of the tetragonal lattice is the same as the [12] direction of the hexagonal lattice.

Of the 10 conjugacy classes obtained from the hexagonal holohedry, the two classes of point-group type $m$ as well as the two classes of type $3 m$ are conjugate under the non-crystallographic rotation by $2 \pi / 12$ which relates the [10] and [21] hexagonal directions. There are 4 new geometric crystal classes obtained from the hexagonal holohedry, so that the total number of geometric crystal classes (and thus also of point-group types) in $\boldsymbol{V}^{2}$ is 10 , as is well known.

\section{Subgroups of the cubic holohedry of $V^{3}$}

We start with the cubic holohedry described with respect to its conventional basis $\left(a=b=c ; \alpha=\beta=\gamma=90^{\circ}\right)$. First taking the subgroups generated by a single one of the 48 symmetry operations and then iteratively adding a further generator to the groups obtained results in 98 different subgroups (including the holohedry; see also [8], page 63). These groups fall into 33 conjugacy classes under the action of the holohedry $\mathcal{P}=m \overline{3} m$ (Table 3 ).

For example, by taking $2_{[100]}$ as generator, we obtain a point group consisting of the identity and a two-fold rotation about the $a$ axis. If we take this as the operation $p$ in Eq. (1) and conjugate it by all the 48 operations of $\mathcal{P}$, we obtain $2_{[100]}, 2_{[010]}$ and $2_{[001]}$, each 16 times. The groups generated by these elements are therefore three different point groups of the same type (point-group type 2) which belong to one conjugacy class. If then we repeat the same procedure with $p=2_{[110]}$, the result is different and consists of 8 occurrences each of $2_{[110]}, 2_{[1 \overline{1} 0]}, 2_{[101]}, 2_{[10 \overline{1}]}$, $2_{[011]}, 2_{[01 \overline{1}]}$. These are six different point groups, which however are again of the same type 2 (containing only a two-fold rotation besides the identity) as those derived before, but belong to a different conjugacy class.

Analogously to the tetragonal case in $V^{2}$, the point groups in these two conjugacy classes belong to the same
Table 3. Conjugacy classes of subgroups of the cubic holohedry $m \overline{3} m$ in $V^{3}$. The group numbering is compatible with the group orders, i.e. if $k>i$ then the group no. $k$ has equal or higher order than the group no. $i$.

\begin{tabular}{|c|c|c|c|c|c|}
\hline $\begin{array}{l}\text { Class } \\
\text { type }\end{array}$ & Order & $\begin{array}{l}\text { Isomorphism } \\
\text { type }\end{array}$ & $\begin{array}{l}\text { Class } \\
\text { Length }\end{array}$ & Generators & $\begin{array}{l}\text { Group } \\
\text { No. }\end{array}$ \\
\hline 1 & 1 & $C_{1}$ & 1 & 1 & 1 \\
\hline$\overline{\mathbf{1}}$ & \multirow[t]{2}{*}{2} & \multirow[t]{5}{*}{$C_{2}$} & 1 & $\overline{1}$ & 2 \\
\hline 2 & & & 3 & $\begin{array}{l}2_{[100]} \\
2_{[010]} \\
2_{[001]}\end{array}$ & $\begin{array}{l}3 \\
4 \\
5\end{array}$ \\
\hline 2 & & & 6 & $\begin{array}{l}2_{[110]} \\
2_{[1 \overline{1} 0]} \\
2_{[101]} \\
2_{[10 \overline{1}]} \\
2_{[011]} \\
2_{[01 \overline{1}]}\end{array}$ & $\begin{array}{l}6 \\
7 \\
8 \\
9 \\
10 \\
11\end{array}$ \\
\hline$m$ & & & 3 & $\begin{array}{l}m_{[100]} \\
m_{[010]} \\
m_{[001]}\end{array}$ & $\begin{array}{l}12 \\
13 \\
14\end{array}$ \\
\hline$m$ & & & 6 & $\begin{array}{l}m_{[110]} \\
m_{[1 \overline{1} 0]} \\
m_{[101]} \\
m_{[10 \overline{1}]} \\
m_{[011]} \\
m_{[01 \overline{1}]}\end{array}$ & $\begin{array}{l}15 \\
16 \\
17 \\
18 \\
19 \\
20\end{array}$ \\
\hline 3 & 3 & $C_{3}$ & 4 & $\begin{array}{l}3_{[111]} \\
3_{[\overline{1} 11]} \\
3_{[1 \overline{1} 1]} \\
3_{[\overline{1} 11]}\end{array}$ & $\begin{array}{l}21 \\
22 \\
23 \\
24\end{array}$ \\
\hline
\end{tabular}


Table 3. Continued.

\begin{tabular}{|c|c|c|c|c|c|c|c|c|c|c|c|}
\hline $\begin{array}{l}\text { Class } \\
\text { type }\end{array}$ & Order & $\begin{array}{l}\text { Isomorphism } \\
\text { type }\end{array}$ & $\begin{array}{l}\text { Class } \\
\text { Length }\end{array}$ & Generators & $\begin{array}{l}\text { Group } \\
\text { No. }\end{array}$ & $\begin{array}{l}\text { Class } \\
\text { type }\end{array}$ & Order & $\begin{array}{l}\text { Isomorphism } \\
\text { type }\end{array}$ & $\begin{array}{l}\text { Class } \\
\text { Length }\end{array}$ & Generators & $\begin{array}{l}\text { Group } \\
\text { No. }\end{array}$ \\
\hline 222 & \multirow[t]{24}{*}{4} & \multirow[t]{24}{*}{$C_{2} \times C_{2}$} & 1 & $2_{[100]}, 2_{[010]}$ & 25 & $\mathrm{mmm}$ & \multirow[t]{5}{*}{8} & \multirow[t]{5}{*}{$C_{2} \times C_{2} \times C_{2}$} & 1 & \multirow{2}{*}{$\begin{array}{l}m_{[100]}, m_{[010]} \\
m_{[001]}\end{array}$} & \multirow[t]{2}{*}{68} \\
\hline \multirow[t]{3}{*}{222} & & & \multirow[t]{3}{*}{3} & $2_{[110]}, 2_{[001]}$ & 26 & \multirow{4}{*}{$\overline{m m m}$} & & & & & \\
\hline & & & & $2_{[101]}, 2_{[010]}$ & 27 & & & & 3 & $m_{[100]}, m_{[011]}, \overline{1}$ & 69 \\
\hline & & & & $2_{[011]}, 2_{[100]}$ & 28 & & & & & $m_{[010]}, m_{[101]}, \overline{1}$ & 70 \\
\hline \multirow[t]{3}{*}{$2 / m$} & & & \multirow[t]{3}{*}{3} & $m_{[100]}, \overline{1}$ & 29 & & & & & $m_{[001]}, m_{[110]}, \overline{1}$ & 71 \\
\hline & & & & $m_{[010]}, \overline{1}$ & 30 & \multirow[t]{3}{*}{$4 / m$} & \multirow[t]{3}{*}{8} & \multirow[t]{3}{*}{$C_{4} \times C_{2}$} & \multirow[t]{3}{*}{3} & $4_{[100]}, m_{[100]}$ & 72 \\
\hline & & & & $m_{[001]}, \overline{1}$ & 31 & & & & & $4_{[010]}, m_{[010]}$ & 73 \\
\hline \multirow[t]{6}{*}{$2 / m$} & & & \multirow[t]{6}{*}{6} & $m_{[110]}, \overline{1}$ & 32 & & & & & $4_{[001]}, m_{[001]}$ & 74 \\
\hline & & & & $m_{[1 \overline{1} 0]}, \overline{1}$ & 33 & \multirow[t]{3}{*}{422} & \multirow[t]{12}{*}{8} & \multirow[t]{12}{*}{$D_{4}$} & 3 & $4_{[100]}, 2_{[010]}$ & 75 \\
\hline & & & & $m_{[101]}, \overline{1}$ & 34 & & & & & $4_{[010]}, 2_{[001]}$ & 76 \\
\hline & & & & $m_{[10 \overline{1}]}, \overline{1}$ & 35 & & & & & $4_{[001]}, 2_{[100]}$ & 77 \\
\hline & & & & $m_{[011]}, \overline{1}$ & 36 & \multirow[t]{3}{*}{$4 m m$} & & & 3 & $4_{[100]}, m_{[010]}$ & 78 \\
\hline & & & & $m_{[01 \overline{1}]}, \overline{1}$ & 37 & & & & & $4_{[010]}, m_{[001]}$ & 79 \\
\hline \multirow[t]{3}{*}{$m m 2$} & & & \multirow[t]{3}{*}{3} & \multirow{3}{*}{$\begin{array}{l}m_{[100]}, m_{[010]} \\
m_{[010]}, m_{[001]} \\
m_{[001]}, m_{[100]}\end{array}$} & \multirow[t]{3}{*}{38} & & & & & $4_{[001]}, m_{[100]}$ & 80 \\
\hline & & & & & & \multirow[t]{3}{*}{$\overline{4} 2 m$} & & & 3 & $\overline{4}_{[100]}, m_{[011]}$ & 81 \\
\hline & & & & & & & & & & $\overline{4}_{[010]}, m_{[101]}$ & 82 \\
\hline \multirow[t]{3}{*}{$m m 2$} & & & 3 & $m_{[110]}, m_{[1 \overline{1} 0]}$ & 41 & & & & & $\overline{4}_{[001]}, m_{[110]}$ & 83 \\
\hline & & & & $m_{[101]}, m_{[10 \overline{1}]}$ & 42 & $\overline{4} m 2$ & & & 3 & $\overline{4}_{[100]}, m_{[010]}$ & 84 \\
\hline & & & & $m_{[011]}, m_{[01 \overline{1}]}$ & 43 & & & & & $\overline{4}_{[010]}, m_{[001]}$ & 85 \\
\hline$m m 2$ & & & 6 & $m_{[100]}, m_{[011]}$ & 44 & & & & & $\overline{4}_{[001]}, m_{[100]}$ & 86 \\
\hline & & & & $m_{[100]}, m_{[01 \overline{1}]}$ & 45 & 23 & 12 & $A_{4}$ & 1 & $3_{[111]}, 2_{[100]}$ & 87 \\
\hline & & & & $m_{[010]}, m_{[101]}$ & $\begin{array}{l}46 \\
47\end{array}$ & $\overline{3} m$ & 12 & $D_{6}$ & 4 & $3_{[111]}, m_{[1 \overline{1} 0]}, \overline{1}$ & 88 \\
\hline & & & & $\begin{array}{l}m_{[010]}, m_{[101]} \\
m_{[001]}, m_{[110]}\end{array}$ & 48 & & & & & $3_{[\overline{1} 11]}, m_{[110]}, \overline{1}$ & 89 \\
\hline & & & & $m_{[001]}, m_{[110]}$ & 49 & & & & & $3_{[1 \overline{1} 1]}, m_{[110]}, 1$ & 90 \\
\hline 4 & 4 & $C_{4}$ & 3 & $4_{[100]}$ & 50 & & & & & $3_{[\overline{1} \overline{1} 1]}, m_{[1 \overline{1} 0]}, \overline{1}$ & 91 \\
\hline & & & & $4_{[010]}$ & 51 & $4 / \mathrm{mmm}$ & 16 & $D_{4} \times C_{2}$ & 3 & $4_{[100]}, m_{[011]}, \overline{1}$ & 92 \\
\hline & & & & $4_{[001]}$ & 52 & & & & & $4_{[010]}, m_{[101]}, \overline{1}$ & 93 \\
\hline$\overline{\overline{4}}$ & & & 3 & $\overline{4}_{[100]}$ & 53 & & & & & $4_{[001]}, m_{[110]}, \overline{1}$ & 94 \\
\hline & & & & $\overline{4}_{[010]}$ & 54 & $\overline{m \overline{\mathbf{3}}}$ & 24 & $A_{4} \times C_{2}$ & 1 & $3_{[111]}, m_{[100]}$ & 95 \\
\hline & & & & & 55 & 432 & 24 & $S_{4}$ & 1 & $4_{[100]}, 3_{[111]}$ & 96 \\
\hline 32 & 6 & D3 & 4 & $3_{[111]}, 2_{[1 \overline{1} 0]}$ & 56 & $\overline{\mathbf{4 3 m}}$ & & & 1 & $\overline{4}_{[100]}, 3_{[111]}$ & 97 \\
\hline & & & & $\begin{array}{l}3_{[\overline{1} 11]}, 2_{[110]} \\
3_{[1 \overline{1} 1]}, 2_{[110]}\end{array}$ & $\begin{array}{l}57 \\
58\end{array}$ & $\overline{m \overline{\mathbf{3}} m}$ & 48 & $S_{4} \times C_{2}$ & 1 & $\begin{array}{l}3_{[111]}, m_{[100]}, \\
m_{[110]},\end{array}$ & 98 \\
\hline
\end{tabular}

point-group type, because the non-crystallographic rotation by $2 \pi / 8$ about the [001] axis maps the lattice direction [100] to [110] and thus conjugates the point group generated by $2_{[100]}$ to that generated by $2_{[110]}$. Neglecting the different orientations in space and considering only the nature of the symmetry operations, the nine groups from these two conjugacy classes are merged into a single geometric crystal class (of type 2). Considering all conjugacy relations by these non-crystallographic rotations, the 33 conjugacy classes of subgroups fall into 25 geometric crystal classes. 


\section{Subgroups of the hexagonal holohedry of $V^{3}$}

By proceeding in the same way as for the cubic holohedry, but this time with respect to the conventional hexagonal basis $\left(a=b ; c ; \alpha=\beta=90^{\circ} ; \gamma=120^{\circ}\right)$ we obtain 54 subgroups of the hexagonal holohedry (including the holohedry), which fall into 32 conjugacy classes (Table 4). However, not all these 54 groups are really new: some of them can be brought to coincide with corresponding subgroups of the cubic holohedry, In order to compare the subgroups obtained from the two holohedries, a common axial setting must be established. This can be done in two different ways: 1) to take the (001) planes of two holohedries in common, giving priority to the monoclinic and orthorhombic subgroups of the two holohedries; 2) to take one of the $\langle 111\rangle$ cubic directions coincident with the [001] hexagonal direction, giving priority to the trigonal subgroups of the two holohedries. The result in terms of number of independent groups is obviously the same. In Table 4 we present the result obtained when the first way is chosen, which represent the natural extension of the case of the tetragonal vs. hexagonal holohedries in $V^{2}$ : two subgroups of type $m m m$ coincide with each other and also the subgroups of this group of type $\mathrm{mmm}$ coincide. The final result is that 16 of the 54 groups for the hexagonal holohedry have already been obtained as subgroups of the cubic holohedry which leads to a total of 136 point groups in $\boldsymbol{V}^{3}$. If instead the trigonal subgroups would have given priority, since $\overline{3} m$ has 16 subgroups (including the full group), this would also have resulted in 16 common subgroups, namely the groups in Table 4 with numbers: $=1,=2,=3,99,100,=12,103,104,107$, =29, 110, $111,120,122,124,130$.

Point groups of the same type but in different conjugacy classes differ for the orientation in space of their symmetry elements; they are conjugate under a non-crystallographic rotation by $2 \pi / 12$, which relates the $\langle 100\rangle$ and $\langle 210\rangle$ hexagonal directions. This is the case for $3 m$, $32, \overline{3} m$, as well as for $\overline{6} 2 m$ vs. $\overline{6} m 2$.

Point groups of the same type occurring in conjugacy classes of the two holohedries are conjugated by a rational matrix relating hexagonal and cubic directions. This is the case for trigonal subgroups of the hexagonal holohedry, which are conjugated to trigonal subgroups of the cubic holohedry via a real matrix relating the hexagonal [001] direction and the cubic $\langle 111\rangle$ directions.

When all these conjugacy relations are taken into account, the 32 conjugacy classes of the hexagonal holohedry give rise to 7 new geometric crystal classes. These, together with the 25 geometric crystal classes obtained from the cubic holohedry, lead to the well-known total to 32 geometric crystal classes (point-group types) in $\boldsymbol{V}^{3}$.

\section{Isomorphism classes}

When point groups are classified in terms of their multiplication table as abstract groups, some groups of the same order may be grouped in one isomorphism class. Groups in the same isomorphism class have the same structure, independently from the elements (symmetry operations) of the groups. For example, groups of type $2 / m$,
Table 4. Conjugacy classes of subgroups of the hexagonal holohedry $6 / \mathrm{mmm}$ in $\mathbf{V}^{3}$. The comparison with the subgroups of the cubic holohedry has been done by taking the (001) planes in common: group numbers of the form " $=k$ " indicate that the respective group is equal to the subgroup no. $k$ of the cubic holohedry.

\begin{tabular}{|c|c|c|c|c|c|}
\hline $\begin{array}{l}\text { Class } \\
\text { type }\end{array}$ & Order & $\begin{array}{l}\text { Isomorphism } \\
\text { type }\end{array}$ & $\begin{array}{l}\text { Class } \\
\text { length }\end{array}$ & Generators & $\begin{array}{l}\text { Group } \\
\text { No. }\end{array}$ \\
\hline 1 & 1 & $C_{1}$ & 1 & 1 & $=1$ \\
\hline$\overline{\mathbf{1}}$ & \multirow[t]{7}{*}{2} & \multirow[t]{7}{*}{$C_{2}$} & 1 & $\overline{1}$ & $=2$ \\
\hline 2 & & & 1 & $2_{[001]}$ & $=5$ \\
\hline 2 & & & 3 & $\begin{array}{l}2_{[100]} \\
2_{[010]} \\
2_{[110]}\end{array}$ & $\begin{array}{l}=3 \\
99 \\
100\end{array}$ \\
\hline 2 & & & 3 & $\begin{array}{l}2_{[120]} \\
2_{[210]} \\
2_{[1 \overline{1} 0]}\end{array}$ & $\begin{array}{l}=4 \\
101 \\
102 \\
\end{array}$ \\
\hline$m$ & & & 1 & $m_{[001]}$ & $=14$ \\
\hline$m$ & & & 3 & $\begin{array}{l}m_{[100]} \\
m_{[010]} \\
m_{[110]}\end{array}$ & $\begin{array}{l}=12 \\
103 \\
104\end{array}$ \\
\hline$m$ & & & 3 & $\begin{array}{l}m_{[120]} \\
m_{[210]} \\
m_{[1 \overline{1} 0]}\end{array}$ & $\begin{array}{l}=13 \\
105 \\
106\end{array}$ \\
\hline 3 & 3 & $C_{3}$ & 1 & $3_{[001]}$ & 107 \\
\hline 222 & \multirow[t]{7}{*}{4} & \multirow[t]{7}{*}{$C_{2} \times C_{2}$} & 3 & $\begin{array}{l}2_{[001]}, 2_{[100]} \\
2_{[001]}, 2_{[010]} \\
2_{[001]}, 2_{[110]}\end{array}$ & $\begin{array}{l}=25 \\
108 \\
109\end{array}$ \\
\hline $2 / m$ & & & 1 & $2_{[001]}, \overline{1}$ & $=31$ \\
\hline $2 / m$ & & & 3 & $\begin{array}{l}2_{[100]}, \overline{1} \\
2_{[010]}, \overline{1} \\
2_{[110]}, \overline{1}\end{array}$ & $\begin{array}{l}=29 \\
110 \\
111\end{array}$ \\
\hline $2 / m$ & & & 3 & $\begin{array}{l}2_{[120],}, \overline{1} \\
2_{[210]}, \overline{1} \\
2_{[11 \overline{1} 0]}, \overline{1}\end{array}$ & $\begin{array}{l}=30 \\
112 \\
113\end{array}$ \\
\hline$m m 2$ & & & 3 & $\begin{array}{l}m_{[100]}, m_{[001]} \\
m_{[010]}, m_{[001]} \\
m_{[110]}, m_{[001]}\end{array}$ & $\begin{array}{l}=40 \\
114 \\
115\end{array}$ \\
\hline$m m 2$ & & & 3 & $\begin{array}{l}m_{[120]}, m_{[001]} \\
m_{[210]}, m_{[001]} \\
m_{[110]}, m_{[001]}\end{array}$ & $\begin{array}{l}=39 \\
116 \\
117\end{array}$ \\
\hline$m m 2$ & & & 3 & $\begin{array}{l}m_{[100]}, m_{[120]} \\
m_{[010]}, m_{[210]} \\
m_{[110]}, m_{[1 \overline{1} 0]}\end{array}$ & $\begin{array}{l}=38 \\
118 \\
119\end{array}$ \\
\hline 32 & \multirow[t]{4}{*}{6} & \multirow[t]{4}{*}{$D_{3}$} & 1 & $3_{[001]}, 2_{[100]}$ & 120 \\
\hline 32 & & & 1 & $3_{[001]}, 2_{[120]}$ & 121 \\
\hline $3 m$ & & & 1 & $3_{[001]}, m_{[100]}$ & 122 \\
\hline $3 m$ & & & 1 & $3_{[001]}, m_{[120]}$ & 123 \\
\hline
\end{tabular}


Table 4. Continued.

\begin{tabular}{|c|c|c|c|c|c|}
\hline $\begin{array}{l}\text { Class } \\
\text { type }\end{array}$ & Order & $\begin{array}{l}\text { Isomorphism } \\
\text { type }\end{array}$ & $\begin{array}{l}\text { Class } \\
\text { length }\end{array}$ & Generators & $\begin{array}{l}\text { Group } \\
\text { No. }\end{array}$ \\
\hline$\overline{\mathbf{3}}$ & \multirow[t]{3}{*}{6} & \multirow[t]{3}{*}{$C_{6}$} & 1 & $3_{[001]}, \overline{1}$ & 124 \\
\hline 6 & & & 1 & $6_{[001]}$ & 125 \\
\hline$\overline{\mathbf{6}}$ & & & 1 & $3_{[001]}, m_{[001]}$ & 126 \\
\hline $\mathrm{mmm}$ & 8 & $C_{2} \times C_{2} \times C_{2}$ & 3 & $\begin{array}{l}m_{[100]}, m_{[120]}, \\
m_{[001]} \\
m_{[010]}, m_{[210]}, \\
m_{[001]} \\
m_{[110]}, m_{[1 \overline{1} 0]}, \\
m_{[001]}\end{array}$ & $\begin{array}{l}=68 \\
127 \\
128\end{array}$ \\
\hline $6 / m$ & 12 & $C_{6} \times C_{2}$ & 1 & $6_{[001]}, m_{[001]}$ & 129 \\
\hline$\overline{3} m$ & \multirow[t]{6}{*}{12} & \multirow[t]{6}{*}{$D_{6}$} & 1 & $3_{[001]}, m_{[100]}, \overline{1}$ & 130 \\
\hline$\overline{3} m$ & & & 1 & $3_{[001]}, m_{[120]}, \overline{1}$ & 131 \\
\hline 622 & & & 1 & $6_{[001]}, 2_{[100]}$ & 132 \\
\hline $6 m m$ & & & 1 & $6_{[001]}, m_{[100]}$ & 133 \\
\hline$\overline{6} 2 m$ & & & 1 & $\begin{array}{l}3_{[001]}, m_{[001]}, \\
m_{[100]}\end{array}$ & 134 \\
\hline $6 m 2$ & & & 1 & $\begin{array}{l}3_{[001]}, m_{[001]}, \\
m_{[120]}\end{array}$ & 135 \\
\hline $6 / \mathrm{mmm}$ & 24 & $D_{6} \times C_{2}$ & 1 & $\begin{array}{l}6_{[001]}, m_{[001]}, \\
m_{[100]}\end{array}$ & 136 \\
\hline
\end{tabular}

222 and $m m 2$ all have the same structure when one forgets about the concrete elements (symmetry operations) in each of them and replaces these by abstract representatives $\mathrm{A}, \mathrm{B}, \mathrm{C}, \mathrm{D}$, such as given in the following table:

\begin{tabular}{lllll}
\hline & $\mathrm{A}$ & $\mathrm{B}$ & $\mathrm{C}$ & $\mathrm{D}$ \\
\hline $2 / m$ & 1 & $2_{[010]}$ & $m_{[010]}$ & $\overline{1}$ \\
222 & 1 & $2_{[010]}$ & $2_{[100]}$ & $2_{[001]}$ \\
$m m 2$ & 1 & $m_{[100]}$ & $m_{[010]}$ & $2_{[001]}$ \\
\hline
\end{tabular}

In terms of these abstract elements, all three groups have the same multiplication table and are thus isomorphic. The 21 (in $\boldsymbol{V}^{2}$ ) and 136 (in $\boldsymbol{V}^{3}$ ) point groups are finally classified into 9 and 18 isomorphism classes, respectively (see also ITA, page 762).

\section{Point groups and point-group types in point space}

Point groups in point space occur as:

1. the symmetry groups of atomic groups and coordination polyhedra;

2. site-symmetry groups (stabilizers of a Wyckoff position).

The former are symmetry groups of finite objects whose inherent symmetry (eigensymmetry) is not restricted to be crystallographic and coincide with the symmetry groups of molecules. Therefore, in this case the number of point groups, but also of point-group types, is infinite. ITA (pages 796-799) call this infinite set of crystallographic and non-crystallographic point groups general point groups and classify them into $8\left(E^{2}\right)$ and $33\left(E^{3}\right)$ general classes, belonging to $3\left(E^{2}\right)$ and $7\left(E^{3}\right)$ general systems, respectively. In $E^{3}$, three of the general systems consist of the point groups with a single principal $n$-fold axis, where $n=2 N+1,4 N, 4 N+2$, the other four are the cubic, icosahedral, cylindrical and spherical systems (ITA Tables 10.1.4.1 and 10.1.4.2).

For atomic groups or coordination polyhedra whose inherent symmetry is crystallographic, point groups in point space are isomorphic to point groups in vector space and this results in a duality between the corresponding forms, i.e. point forms (in point space) and face forms (in vector space) (ITA, page 764). For example, the face form trigonal dipyramid has eigensymmetry $\overline{6} 2 \mathrm{~m}$; its dual in point space (point form) is a trigonal antiprism.

Site-symmetry groups (see also [13]) are subgroups of space groups and thus obey the crystallographic restriction and are always finite groups. But apart from the space groups without special positions $\left(1, p g\right.$ in $E^{2}$ and $1, P 2_{1}$, $P c, C c, P 2_{1} 2_{1} 2_{1}, P c a 2_{1}, P n a 2_{1}, P 4_{1}, P 4_{3}, P 3_{1}, P 3_{2}, P 6_{1}$, $\mathrm{PG}_{5}$ in $E^{3}$ ) each space group $\mathcal{G}$ contains infinitely many site-symmetry groups, due to the conjugation by the infinite translation subgroup.

The site-symmetry groups are classified into geometric crystal classes exactly as point groups are; there are thus $10\left(E^{2}\right)$ and $32\left(E^{3}\right)$ types of site-symmetry groups. Sitesymmetry groups are however oriented groups: two groups of the same type may differ by their orientation, and this implicitly calls for a finer classification ${ }^{6}$. There is a partial correspondence between site-symmetry groups of the same type and with the same orientation and face forms with the same inherent symmetry and the same orientation. The latter are classified in up to three orders. Although the correspondence is only partial, site-symmetry groups can be classified in the same way. However, the term "order" is profoundly established for the number of elements (symmetry operations, in our case) in a group and thus extending the classical term "order" for the classification of face forms to the classification of site-symmetry groups would call for misunderstandings. In the French literature, the word espèce is used; its corresponding English word species seems a good compromise. We therefore propose to classify site-symmetry groups of the same type and with the same orientation into the same site-symmetry group species.

There is evidently a $1-1$ correspondence between species of site-symmetry groups and point groups in vector space, from which we conclude easily about the existence of 21 site-symmetry group species in $E^{2}$ and 136 in $E^{3}$.

At first glance, the concept of species of site-symmetry groups may seem to be related to that of Wyckoff positions or Wyckoff sets, but it can be easily shown that this is not the case.

Recall that for a space group $\mathcal{G}$, the affine normalizer $\mathcal{N}_{\mathcal{A}}(\mathcal{G})$ of $\mathcal{G}$ is defined to consist of all elements in the

6 In part, this finer classification can actually be read off from the symbols given for the site symmetry in ITA, since these symbols are more verbose than those for the point groups. 
group $\mathcal{A}$ of affine mappings ${ }^{7}$ that map $\mathcal{G}$ onto itself by conjugation. Two elements of $\mathcal{G}$ which are conjugate by an element of $\mathcal{N}_{\mathcal{A}}(\mathcal{G})$ play equivalent roles as symmetry operations and two points of $E^{n}$ which are mapped to each other under $\mathcal{N}_{\mathcal{A}}(\mathcal{G})$ are geometrically indistinguishable.

Any Wyckoff position of $\mathcal{G}$ is transformed onto itself by all elements of $\mathcal{G}$, but not necessarily by the elements of $\mathcal{N}_{\mathcal{A}}(\mathcal{G})$. Wyckoff positions that are inter-transformed by $\mathcal{N}_{\mathcal{A}}(\mathcal{G})$ are defined to belong to the same Wyckoff set.

Site-symmetry groups for positions of the same Wyckoff set clearly are of the same type, but not necessarily of the same species, because a Wyckoff set may contain Wyckoff positions whose site-symmetry groups are differently oriented. For example, in P222 the 12 Wyckoff positions $2 i$ to $2 t$ with site-symmetry group of type 2 belong to the same Wyckoff set. These site-symmetry groups fall into three species with different orientations, each corresponding to four Wyckoff positions: 2 .. for positions $2 i$ to $2 l, .2$. for positions $2 m$ to $2 p$ and. .2 for positions $2 q$ to $2 t$. In general, however, the partition into species is not just a subdivision of the partition into Wyckoff sets, as is illustrated by the following example. In C222 there are seven Wyckoff positions with site-symmetry group of type 2: $4 e$ to $4 k$. These are divided into three Wyckoff sets: $4 e$ to $4 h, 4 i, 4 j$ and $4 k$. While the first Wyckoff set splits into two species, the other two Wyckoff sets belong to the same species:

\begin{tabular}{llllllll}
\hline $\begin{array}{l}\text { Wyckoff } \\
\text { sets }\end{array}$ & $(4 e$ & $4 f$ & $4 g$ & $4 h)$ & $(4 i)$ & $(4 j$ & $4 k)$ \\
\hline $\begin{array}{l}\text { site } \\
\text { symmetry }\end{array}$ & $2 .$. & $2 .$. & .2. & .2. &. .2 &. .2 &. .2 \\
\hline
\end{tabular}

But even in a single Wyckoff position the site-symmetry groups may be differently oriented. In $P 422$, position $4 l$ with oriented site-symmetry symbol .2 . contains both the coordinates $x, 0,0$ and $0, x, 0$. The site-symmetry group for the first position is generated by a twofold rotation around the $a$-axis, the site-symmetry group of the second by a twofold rotation around the $b$-axis. Clearly, the two site-symmetry groups are conjugate in the space group, but they are differently oriented point groups.

\section{Point groups and factor groups: relation with space groups}

The ITA (page 725) establish a further isomorphism between the (vector) point group of the crystal and the linear parts of the matrices representing the symmetry operations of a space group. We conclude our analysis by giving some details on this isomorphism, because it implies a further correspondence between $E^{n}$ and $\boldsymbol{V}^{n}$.

The set of translations representing the lattice of a crystal pattern is an infinite group called the translation sub-

\footnotetext{
7 An affine mapping is any mapping that preserves collinearity and ratios of distances, while angles and lengths in general are not kept. When these are preserved too, one speaks of a Euclidean mapping, a special case of an affine mapping.
}

group $\mathcal{T}$ of the space group $\mathcal{G}$. The translation subgroup $\mathcal{T}$ is a normal subgroup of $\mathcal{G}$, which means that for any translation $t$ in $\mathcal{T}$ all its conjugate elements also belong to $\mathcal{T}$, i.e. for an arbitrary element $g$ in $\mathcal{G}$ the conjugation relation (1) reads as $\mathrm{gtg}^{-1}=t^{\prime}$ for some translation $t^{\prime}$ in $\mathcal{T}$.

The coset decomposition of $\mathcal{G}$ with respect to $\mathcal{T}$ is a partition of $\mathcal{G}$ into a finite number $m$ of cosets. Each coset is an infinite set of the form

$$
W T=\{W t \mid t \in T\}=\{t W \mid t \in T\}=T W
$$

where the second equality is due to the fact that $\mathcal{T}$ is a normal subgroup ${ }^{8}$. Choosing one symmetry operation $W_{i}$ $(i=1, \ldots, m)$ from each coset (assuming $W_{1}$ to be the identity of $\mathcal{G}$ ) gives the coset decomposition

$$
\begin{aligned}
\mathcal{G} & =\mathcal{T} W_{1} \cup \mathcal{T} W_{2} \cup \ldots \cup \mathcal{T} W_{i} \cup \ldots \cup \mathcal{T} W_{m} \\
& =W_{1} \mathcal{T} \cup W_{2} \mathcal{T} \cup \ldots \cup W_{i} \mathcal{T} \cup \ldots \cup W_{m} \mathcal{T} .
\end{aligned}
$$

With respect to a given basis of $E^{n}$, a symmetry operation $\mathrm{W}_{i}$ can be written as a matrix $\boldsymbol{W}$, called the linear part (representing the rotational component of the operation) and a vector $\boldsymbol{w}$ called the vector part (representing the translational component of the operation). Space groups whose coset decomposition (3) result in the same set of matrices $\boldsymbol{W}$ belong to the same geometric crystal class and differ in their vector parts $\boldsymbol{w}$.

The set of matrices $\boldsymbol{W}$ forms a group $\mathcal{W}$ acting on $E^{n}$ that is isomorphic to the point group $\mathcal{P}$ which acts on $\boldsymbol{V}^{n}$. The number $m$ of cosets in Eq. (3) is the order of the point group $\mathcal{P}$ and thus also of the group $\mathcal{W}$. It can be easily shown that $\mathcal{W}$ is a symmetry group for the lattice of the crystal pattern.

Furthermore, the group $\mathcal{W}$ of linear parts is also isomorphic to the factor group or quotient group $\mathcal{G} / \mathcal{T}$. The factor group is the set of all cosets of $\mathcal{T}$ in $\mathcal{G}$, i.e.:

$$
\mathcal{G} / \mathcal{T}=\{g \mathcal{T} \mid g \in \mathcal{G}\}
$$

The factor group is a special kind of group in which each element, instead of being a symmetry operation, is a coset with respect to a subgroup. Multiplication of cosets is determined by multiplying representatives, i.e. $g_{1} \mathcal{T} \cdot g_{2} \mathcal{T}=$ $\left(g_{1} g_{2}\right) \mathcal{T}$. Again, the cosets only give rise to a group in this way because $\mathcal{T}$ is a normal subgroup of $\mathcal{G}$.

By taking the products of each element of $\mathcal{W}$ and of $\mathcal{T}$, one builds the so-called semi-direct product of $\mathcal{T}$ by $\mathcal{W}^{9}$. The result is a space group belonging to one of the 13 (in $E^{2}$ ) or 73 (in $E^{3}$ ) symmorphic types. Symmorphic space groups are distinguished by the property that their site-symmetry groups (in $E^{n}$ ) of highest order (lowest multiplicity) are isomorphic to their point groups $\mathcal{P}$ (in $\boldsymbol{V}^{n}$ ). Space groups of non-symmorphic type cannot be obtained as semi-direct product of $\mathcal{T}$ by $\mathcal{W}$ and do not contain any Wyckoff position whose site-symmetry group is isomorphic to $\mathcal{P}$.

\footnotetext{
${ }^{8}$ In general, i.e. for a non-normal subgroup $\mathcal{T}$, the left coset $W \mathcal{T}$ and the right coset $\mathcal{T} W$ are different.

${ }^{9}$ If $\mathcal{W}$ were a normal subgroup, one would obtain a direct pro$d u c t$, but in space groups the (nontrivial) finite subgroups are never normal.
} 


\section{Conclusions}

The concept of point group in crystallography is far more complex than normally considered, because it applies to different objects in two different, dual spaces. The notions of conjugacy and isomorphism allow a useful systematisation of the different notions of point groups. One should, however, be aware that some common terminology is actually imprecise. For example, the expression "point group of the space group" requires an (implicit) identification, since it refers to a group $\mathcal{W}$ (the group of linear parts) which is (in a natural way) isomorphic to the point group $\mathcal{P}$, but does not coincide with it, since $\mathcal{P}$ is defined to be acting on vector space.

Acknowledgments. The first author wishes to thank Prof. Werner Fischer (Marburg University), Prof. Theo Hahn (RWTH Aachen) and Prof. Mois I. Aroyo (Universidad del Pais Vasco, Bilbao) for useful discussions. The comments of two anonymous reviewers are gratefully acknowledged.

\section{References}

[1] Bravais, M.A.: Mémoire sur les systèmes formés par des points distribués régulièrement sur un plan ou dans l'espace. J. École Polytechn., Cahier 33 XIX, (1850) 1-128 (English translation: On The Systems Formed by Points Regularly Distributed on a Plane or in Space, translated by Amos J. Shaler, The Crystallographic Society of America Memoir Number No. 1, 1949).

[2] Brown, H.; Bülow, R.; Neubüser, J.; Wondratschek, H.; Zassenhaus, H.: Crystallographic groups of four-dimensional space. John Wiley, New York, (1978).
[3] Donnay, J. D. H.: The Structural Classification of Crystal Point Symmetries. Acta Crystallogr. A33 (1977) 979-984.

[4] Ewald, P. P.: Zur Theorie der Interferenzen der Röntgentstrahlen in Kristallen. Z. Phys. 14 (1913) 465-472.

[5] Gibbs, J. W.: Vector Analysis. Privately printed, New Haven, CT, (1881). Later printed by Yale University; 1st printing 1901; 9th printing 1947; available at http://www.archive.org/details/ $117714283,476 \mathrm{pp}$.

[6] Grimmer, H.; Nespolo, M.: Geminography - the crystallography of twins. Z. Kristallogr. 221 (2006) 28-50.

[7] Hahn, Th. (Ed.): International Tables for Crystallography Vol. A, 5th edition. D. Dordrecht/Boston: Reidel Publishing Company, (2002).

[8] Hahn, Th.; Wondratschek, H.: Symmetry of Crystals: Introduction to International Tables for Crystallography Vol. A. Sofia: Heron Press, (1994) 144 pp.

[9] Magma: Computational Algebra Group, School of Mathematics and Statistics, University of Sydney. The Magma Computational Algebra System for Algebra, Number Theory and Geometry. (2004) http://magma.maths.usyd.edu.au/magma.

[10] Moses, A. J.; Parsons, C. L.: Elements of mineralogy, crystallography and blowpipe analysis from a practical standpoint, including a description of all common or useful minerals, their formation and occurrence, the tests necessary for their identification. New York: D. Van Nostrand Company, (1897). vii $+342 \mathrm{pp}$.

[11] Nebe, G.: The mathematical background of the subgroup tables. Section 1.5. In: International Tables for Crystallography. Vol. A1, D. Dordrecht/Boston: Reidel Publishing Company, (2004) pp. 29-40.

[12] Rigault, G.: Il reticolo polare di Bravais e il reticolo reciproco. Acc. Sc. Torino Atti Sc. Fis. 133 (1998) 1-8.

[13] Wondratschek, H.: Matrices, Mappings and Crystallographic Symmetry. International Union of Crystallography, Teaching Pamphlet (2002) No. 22, 81 pp. 